\title{
A V-Shape Optical Pin Interface for Board Level Optical Interconnect
}

\author{
N.N.H. Saris, ${ }^{* 1}$ O. Mikami, ${ }^{1}$ A. Hamzah, ${ }^{1}$ S. Ambran, ${ }^{1}$ and C. Fujikawa ${ }^{2}$ \\ ${ }^{I}$ Department of Electronic Systems Engineering, Malaysia-Japan International Institute of Technology (MJIIT) \\ Universiti Teknologi Malaysia, 54100 Jalan Sultan Yahya Petra, Kuala Lumpur, Malaysia \\ ${ }^{2}$ Graduate School of Engineering, Tokai University 4-1-1, Kitakaname, Hiratsuka, Kanagawa, 259-1292 Japan
}

Received December 04, 2018; accepted March 27, 2008; published March 31, 2018

\begin{abstract}
This paper introduces a new interface of an optical pin for Printed Circuit Boards (PCBs), the V-shape cut type which is an innovation from the 90-degree cut type of optical pin. The effectiveness is determined by optical characteristics through OptiCAD and by experiment. The simulation used a model of ray tracing analysis which is a one to two (split) connection function model. For the experiment, a Polymer Optical Fibre (POF) V-shape optical pin has been fabricated. It was found that the $\mathrm{V}$-shaped optical pin has a multi-branched function and is applicable to optical interconnection.
\end{abstract}

The technology for on board level; especially in Printed Circuit Boards (PCBs) has evolved rapidly from copper to optical wiring. Conventional copper wiring is influenceable by crosstalk and skin effects [1, 2]. Even though optical wiring has many advantages over copper wiring, such as insusceptibility to Electromagnetic Interference (EMI), cost-effectiveness, high bandwidth operation and being a density system [3], the optical connection has raised questions about its potential as an easy coupling and fine alignment between optical wiring and optical devices.

To solve this issue, a 90-degree optical path conversion optical pin was introduced as the practical optical interconnect for Opto-electronics (OE) PCB [1, 4, 5]. It has the ability to increase coupling efficiency and tolerance. The optical pin is an important optical device due to its function as an optical connector between optical elements, such as a Vertical Cavity Surface Emitting Laser (VCSEL) or Photodiodes (PD), and optical waveguide [6]. In general, the function of the optical pin can be explained as an optical device which is used to change the direction of propagating light from a vertical to a horizontal path [4].

Figure 1 shows the interface of a 90-degree optical path conversion optical pin; known as a 45-degree optical pin. This optical pin is limited to a single connection and has a unidirectional conversion function. This 45-degree cut type optical pin functions as a splitter for optical path conversion. The 45-degree optical pin interface enables a 90-degree optical path conversion coupling. In the future, it is expected that various multi-directional couplings of optical path conversion and optical path branching

*E-mail: nnhuda3@live.utm.my functions will be implemented in optical interconnect technologies on board level $[1,7]$.

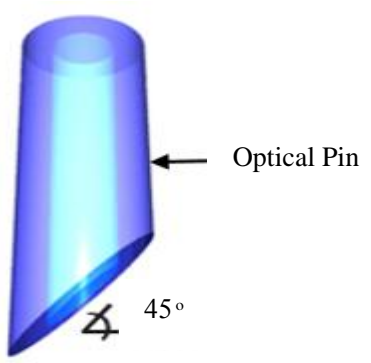

Fig. 1. 45-Degree Cut Type Optical Pin.

However, it is worthwhile to pursue proposing a new architecture of the optical pin interface, the $\mathrm{V}$-shape cut type optical pin. Although the 45-degree cut type optical pin that is described to have a 90-degree optical path conversion function, the $\mathrm{V}$ shaped cut type optical pin has the function of converting incident light into two paths. This is so in addition to another 90-degree optical path conversion function; hence, it is an optical pin that can be branched.

Figures 2 and 3 show the proposed V-shape cut type optical pin and its fabrication method by using a diamond blade with a 90-degree cut at the tip. The tip of the POF can be cut into a $\mathrm{V}$ shape. However, there is a possibility that the processed end may be roughened leading to the blurring of optical fibre. It is optimal to fix the optical pin with a ferrule and cut together with the ferrule. With this method it is possible to obtain an optical end face without polishing. The application of a V-shape optical pin is similar to a conventional 45-degree cut type optical pin that can be applied to OE. The V-shape optical pin is attached to OE Surface Mount Devices (SMD) and then inserted through the hole on the OE-PCBs. The embedded optical waveguide in the PCB provides the holes so that the optical pin can be inserted. 


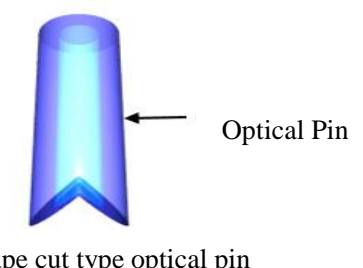

V-shape cut type optical pin

Fig. 2. The V-Shape Cut Type Optical Pin.

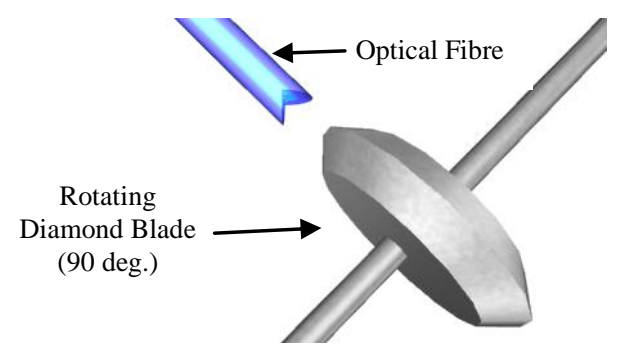

Fig. 3. Fabrication method of a V-shape Cut Type Optical Pin.

In this study, the coupling efficiency and misalignment tolerance between the optical waveguide core and the proposed optical pin (V-shape cut type optical pin) are simulated by employing a ray tracing method analysis by using OptiCAD. In this ray tracing method analysis, a oneto-two (1:2) connection function model (split model) is used. This model applied the rectangular optical waveguide with a core of 50um and a cladding thickness of $40 \mu \mathrm{m}$. The refractive index for the core and cladding are 1.489 and 1.471 respectively. The type of optical pin used was metal coated and rectangular in shape. It has a $50 \mu \mathrm{m}$ core and $37.5 \mu \mathrm{m}$ cladding thickness. While for the VCSEL, it has 10,000 rays of a light beam with $6^{\circ}$ light divergence of half angle and $1 \mathrm{~mm}$ beam spot of half width.

For this split model, the VCSEL is placed on top of the V-shape cut type optical pin between two PDs, PD (1) and PD (2). At the same time, each PD is placed on the top of the 45-degree optical pin. The VCSEL and the PDs are connected through the optical waveguide. The split model applied in this research is shown in Fig. 4. Based on the split model, the position of the $\mathrm{V}$-shape optical pin is at zero Y-axis. The light beams are branched and propagated to the left and right along the optical waveguide. Therefore, both PDs receive light from the 45-degree cut type optical pin. It is determined that, even though the position of the $\mathrm{V}$-shape optical pin deviates in the direction of the Y-axis, the optical power is branched into two directions by half at a time as shown in the analysis in Fig. 5.

The coupling efficiency analysis indicates that the maximum reading recorded for both PD (1) and PD (2) is the same at $-3.4 \mathrm{~dB}$. This result verifies that high efficiency of the V-shape cut type optical pin transmission can be achieved by using a split model in PCB technology. It is very important to determine positional tolerance from the optical coupling efficiency characteristics, as it is practically difficult to make the optical axis of the optical pin completely coincide with the optical axis of the optical waveguide when mounting the V-shape cut type optical pin. Therefore, the tolerance is read with a $1 \mathrm{~dB}$ reduction from the maximum optical coupling efficiency resulting in $\pm 16 \mu \mathrm{m}[8]$.

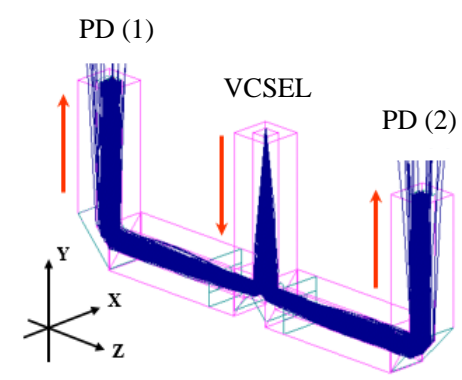

Fig. 4. Ray Tracing Method: Split Model.

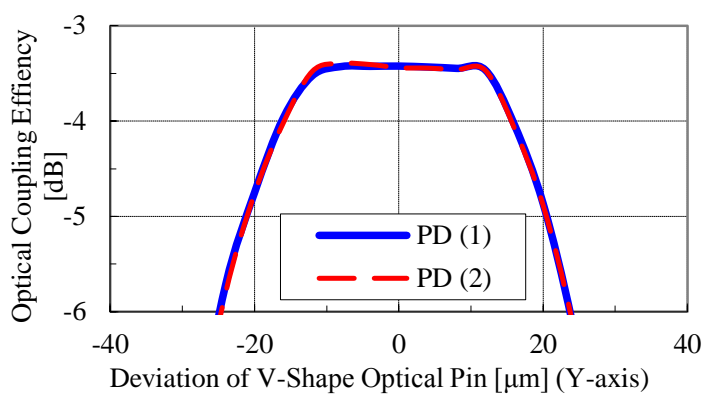

Fig. 5. Split Model Coupling Efficiency of V-shape Cut Type Optical Pin.

In this research, apart from simulation, an experiment using the prototype of a $\mathrm{V}$-shape cut type optical pin is conducted to investigate the basic characteristics of the $\mathrm{V}$-shape cut type optical pin prototype. This prototype of the V-shape cut type optical pin was made by using a 5 centimetre $(\mathrm{cm})$ length of Polymer Optical Fibre (POF). A large POF diameter of $980 \mu \mathrm{m}$ is utilised to ease the fabrication process [9]. The POF used was Super Eska, made by Mitsubishi Rayon, which is widely used as a DAI (Digital Audio Interface) in the audio field. The prototype of the POF V-shape cut type optical pin is shown in Fig. 6.

The arrangement of the POF V-shape cut type optical pin in the experiment is illustrated in Fig. 7. The purpose of this experiment is to investigate the basic optical characteristics of the POF V-shape cut type optical pin. However, the numerical calculation of coupling efficiency and the measured values of this experiment are not compared. This is due to shape difference of the core in simulation and the POF, being rectangular for the simulation and circular for the POF. In this experiment, multi-branched optical pins are designed to branch multimode light into optical paths, so it is essential to determine any influence on this 
branching function based on coupling positions with the light source. A wavelength light of $633 \mathrm{~nm}$ was made incident on 50/125 $\mu \mathrm{m}$ diameter Graded Index Multimode Fibre (GI-MMF) from the Fibre Connector (FC). When the two branched beams were emitted from the V-shaped cut surface, the reception and optical coupling efficiency were measured by using PDs. The maximum light branching efficiency was recorded in Fig. 8.

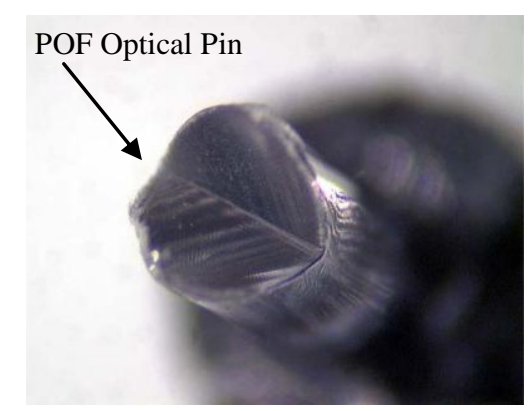

Fig. 6. Prototype of a POF V-shape Cut Type Optical Pin.

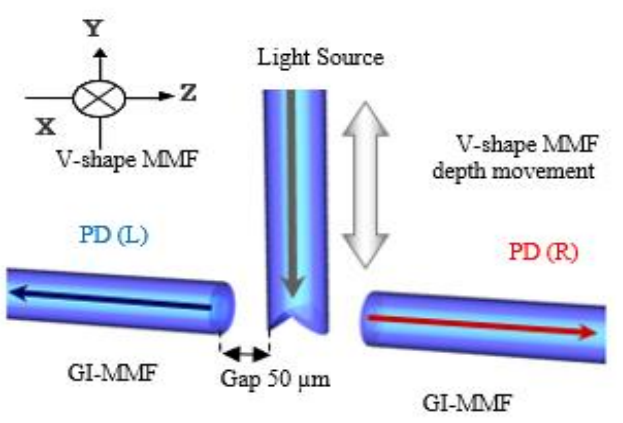

Fig. 7. The Positional Experiment of a POF V-shape Cut Type Optical Pin.

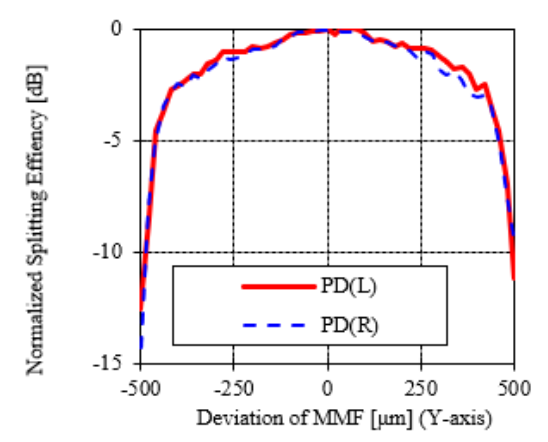

Fig. 8. Positional Tolerance of Optical Pin along Depth Direction.

When the V-shaped cut POF coupled with GI-MMF is shifted in the direction depth, the tolerance width allowing $1 \mathrm{~dB}$ reduction from the maximum, light branching efficiency for PD at the left and right was recorded, both at $\pm 300 \mu \mathrm{m}$. It was found that the light propagated in the $\mathrm{V}$-shape cut type POF was reflected by the V-shaped face and converted to 90-degrees in the left and right directions. However, light does not satisfy the total reflection condition on the V-shaped face because some is generated as transmission. This transmitted light is considered as a loss, but it can be improved by applying metal coating to the cut surface in the future.

Considering that the core diameter of the V-shaped cut type POF is $980 \mu \mathrm{m}$ and a tolerance of $600 \mu \mathrm{m}$ in total in the $\mathrm{Y}$ axis direction is obtained, the above results indicate that within the range of the core diameter from the core centre of the optical pin to be 60 percent. A stable multimode branching function can be achieved if the optical axis to be coupled to the optical fibre is positioned in this range.

If this idea is applied to an MMF type optical pin with a core diameter of $50 \mu \mathrm{m}$, stable branching becomes possible if the optical axis centre of the VCSEL is positioned at a radius of $15 \mu \mathrm{m}$ from the core centre. The tolerance of a radius of $15 \mu \mathrm{m}$ is within the range of being sufficiently mountable. From the above examination, it is possible to provide the multi-mode branching function to an optical interconnection using optical pins.

Based on the experiments conducted, it can be seen that the V-shaped cut type POF is capable of a stable multi-mode branching function. Therefore, it is concluded that simulation by using a split model and analysis of a POF $\mathrm{V}$-shaped cut type optical pin demonstrates that a multi-branching function can be applied to optical pins effectively. It is believed that the evolution of the optical pin interface from 90-degree to an additional angle of 90-degree path conversion of light will lead to substantial advances in optical interconnection in the future.

\section{References}

[1] O. Mikami et al. Optical pin interface for 90-deg optical path conversion coupling to Printed Wiring Board, IEEE TENCON Conf. 2016

[2] C. DeCusatis, "Data center architectures" in Optical Interconnects for Data Centers (Elsevier, 2017), p. 3-41.

[3] M. Duranton, D. Dutoit, S. Menezo, "Key requirements for optical interconnects within data centers" in Optical Interconnects for Data Centers (Elsevier, 2017), p. 75-94.

[4] Y. Itoh et al., J. Japan Instit. Electronics Packaging 4(6), 497 (2001)

[5] T. Uchida, O. Mikami, IEICE Transact. Electron. 80(1), 81 (1997).

[6] I. Papakonstantinou et al., IEEE Transact. Adv. Packaging 31(3), 502 (2008).

[7] K. Nakama, et al., Optical connection device (Google Patents, 2006).

[8] R. Ramaswami, K. Sivarajan, G. Sasaki, Optical networks: a practical perspective (Morgan Kaufmann, 2009).

[9] X.C. Tong, Advanced materials for integrated optical waveguides (Springer, 2014). 